\title{
Caraterização Composicional do AES - Um Copolímero de Enxertia de Poli(Estireno-Co-Acrilonitrila) em Poli(Etileno-co-Propileno-co-Dieno)
}

\author{
Renato Turchet, Maria I. Felisberti \\ Instituto de Química, UNICAMP/SP
}

Resumo: O objetivo deste trabalho é a caracterização do AES, um copolímero de enxertia de poli(estireno-co-acrilonitrila), SAN, em poli(etileno-co-propileno-co-dieno), EPDM. Para tanto, o AES foi submetido à extração seletiva de seus componentes: o SAN livre, o EDPM livre, e o copolímero de enxertia EPDM- $g$-SAN. O AES e suas frações foram caracterizados por espectroscopia de infravermelho, análise elementar, calorimetria diferencial de varredura e ressonância magnética nuclear, $\mathrm{RMN}^{1} \mathrm{H}$ e $\mathrm{RMN}^{13} \mathrm{C}$. O AES analisado apresenta a seguinte composição em massa: 65\% de EPDM-g-SAN, $13 \%$ de EPDM livre e 22\% de SAN livre. O EPDM apresenta 69,8\% em massa de etileno, 26,5\% em massa de propileno e 4,6\% em massa do dieno, 2-etilideno-5-norboneno, ENB. O SAN apresenta razão em massa acrilonitrila/estireno de 28/72 e distribuição randômica de comonômeros de estireno e acrilonitrila. Estes resultados são concordantes com a composição do AES fornecida pelo fabricante, indicando que a metodologia proposta é adequada.

Palavras-chave: Caracterização, copolímero de enxertia, AES, EPDM.

\section{Compositional Characterization of AES - A Graft Copolymer Based on Poly(Styrene-co-Acrylonitrile) and Poly(Etyhlene- co-Propylene-co-Diene)}

\begin{abstract}
This work aims the characterization of AES, a graft copolymer based on poly(styrene-co-acrylonitrile), SAN, and poly(etyhlene-co-propylene-co-diene), EPDM. AES was submitted to selective extraction of its components: free SAN, EPDM chains and the graft copolymer EPDM-g-SAN. AES and its fractions were characterized by infrared spectroscopy, elemental analysis, differential scanning calorimetry, ${ }^{13} \mathrm{C}$ and ${ }^{1} \mathrm{H}$ nuclear magnetic resonance. The AES has $65 \mathrm{wt} \%$ of EPDM- $g$-SAN, $13 \mathrm{wt} \%$ of free EPDM and $22 \mathrm{wt} \%$ of free SAN. EPDM has $69.8 \mathrm{wt} \%$ of ethylene, $26.5 \mathrm{wt} \%$ of propylene and $4.6 \mathrm{wt} \%$ of diene, 2-ethylidene-5-norbonene ENB. SAN presents acrylonitrile/styrene mass ratio of 28/ 72 and a random distribution of acrylonitrile and styrene comonomers. These results are in agreement with the composition reported by the AES supplier, indicating that the proposed methodology is adequate.
\end{abstract}

Keywords: Characterization, graft copolymer, AES; EPDM.

\section{Introdução}

O elastômero termoplástico acrilonitrila-butadienoestireno, ABS, é um dos plásticos de engenharia mais utilizados, principalmente na indústria automobilística, pois apresenta alta resistência ao impacto e rigidez e, juntamente com estas propriedades, fácil obtenção e processabilidade ${ }^{[1,2]}$. No entanto, torna-se amarelado e perde as propriedades mecânicas quando exposto a intempéries, o que limita sua utilização em várias aplicações ${ }^{[3]}$. Esta baixa resistência a intempéries é atribuída ao alto teor de insaturação da fase polibutadieno, a fase dispersa do $\mathrm{ABS}^{[4-8]}$. Uma das propostas para solucionar esta questão é a substituição do polibutadieno por um elastômero com menor teor de insaturações ${ }^{[1,2,8-10]}$. Pesquisas levaram ao terpolímero poli(etileno-co-propileno-co-dieno), EPDM, que apresenta maior resistência à temperatura, à oxidação, à luz, ao oxigênio, ao ozônio e à radiação ultravioleta em comparação com o polibutadieno ${ }^{[4,6,10,11]}$. Esta substituição gerou o AES, que pode ser obtido por copolimerização radicalar de estireno e acrilonitrila em presença de poli(etileno-copropileno-co-dieno), EPDM ${ }^{[9,12]}$. Neste processo, ocorre a copolimerização do estireno e da acrilonitrila, formando o SAN, que encontra-se tanto na forma livre como enxertada no EPDM, sendo que uma parte das cadeias de EPDM também permanece livres de $\mathrm{SAN}^{[12]}$. Neste sistema, o copolímero de enxertia atua como um compatibilizante entre as fases imiscíveis de EPDM e de SAN. Sua estrutura e propriedades variam com as razões molares estireno/ acrilonitrila no SAN, etileno/propileno no EPDM e SAN/EPDM no AES. A ordem de adição dos reagentes, como também as condições de polimerização afetam as

Autor para correspondência: Maria Isabel Felisberti, Grupo de Pesquisa em Polímeros - GPPol, Instituto de Química, Bloco I-102, UNICAMP, Caixa Postal 6154, CEP: 13083-970, Campinas, SP. E-mail: misabel@iqm.unicamp.br 
Tabela 1. Dados fornecidos pelo fabricante para o AES

\begin{tabular}{cccccc}
\hline & $\begin{array}{c}\text { Índice de Fluidez } \\
(\mathbf{g} / \mathbf{1 0} \mathbf{m i n})\end{array}$ & $\begin{array}{c}\text { Densidade } \\
\left(\mathbf{g} / \mathbf{c m}^{\mathbf{3}}\right)\end{array}$ & $\begin{array}{c}\text { Razão } \\
\text { SAN/EPDM }\end{array}$ & \% SAN enxertado & $\begin{array}{c}\text { Razão } \\
\text { Etileno/Propileno }\end{array}$ \\
\hline AES & 20 & 0,98 & $50 / 50$ & 30 & $70 / 30$ \\
\hline
\end{tabular}

propriedades do material obtido ${ }^{[12-14]}$. Devido a estes fatores, o AES é um material complexo, apresentando uma multiplicidade de distribuições de composição química e de massa molar, o que dificulta a sua caracterização ${ }^{[12]}$.

O AES é utilizado na compatibilização de blendas de SAN/ $\mathrm{EPDM}^{[4]}$ e na tenacificação de polímeros como: o poli(tereftalato de butileno $)^{[3]}$, o $\mathrm{SAN}^{[6]}$, a poliamida $6^{[7,13,15,16]}$ e o poli(metacrilato de metila $)^{[17]}$.

Chiantore ${ }^{[12]}$, determinou a composição do AES por cromatografia por exclusão de tamanho, utilizando detectores de índice de refração e de ultravioleta simultaneamente. Contudo, este método resultou em erros experimentais devido a diferenças de sensibilidade dos detectores. Utilizando a cromatografia por precipitação e redissolução, Chiantore ${ }^{[12]}$ mostrou ser possível separar as frações do AES, entretanto, a determinação quantitativa é fortemente influenciada pelos solventes e condições experimentais.

Outro método, mais simples, porém eficiente e que pode ser utilizado na caracterização do AES é a extração seletiva. Neste método, utiliza-se solventes específicos para extrair cada um de seus componentes. Após a extração dos componentes, a composição de cada um, em termos de porcentagem de nitrogênio, carbono e hidrogênio, pode ser determinada por análise elementar. Contudo, além da caracterização composicional, torna-se necessário conhecer a extensão da enxertia, a distribuição de comonômeros e a massa molar, sendo necessária a utilização de várias técnicas analíticas, tais como a ressonância magnética nuclear de hidrogênio e de carbono, espectroscopia na região de infravermelho e calorimetria diferencial de varredura. Sendo assim, este trabalho visa o desenvolvimento de uma metodologia baseada na solubilidade diferenciada dos componentes do AES para uma ampla caracterização deste material.

\section{Experimental}

O AES submetido a separação e caracterização de seus diferentes componentes, SAN livre, EPDM livre e EPDM-gSAN corresponde a um produto comercial, Royaltuf 372P20, fornecido pela Crompton Corporation (Middlebury, USA). A Tabela 1 apresenta as características fornecidas pelo fabricante $^{[18]}$.

O AES foi submetido a extração seletiva, na qual utilizouse solventes específicos para a solubilização de seus componentes.

\section{Extração seletiva}

A extração da fase SAN livre do AES foi realizada com acetona em um extrator Soxlet por $12 \mathrm{~h}$. A solução contendo SAN foi adicionada a um volume de n-hexano 5 vezes maior que a solução em acetona, resultando na precipitação do SAN,

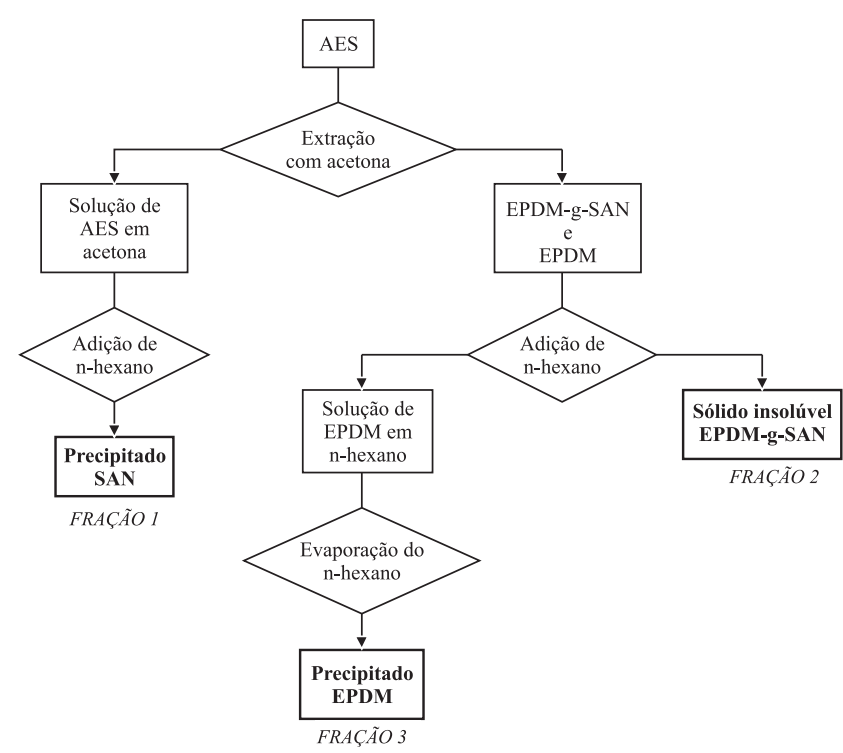

Figura 1. Representação esquemática da extração das frações do AES.

denominada Fração 1, a qual foi isolada, seca e pesada. O resíduo sólido da extração de SAN, que contém o EPDM- $g$-SAN e o EPDM livre, foi suspenso em n-hexano por 48 horas para a extração do EPDM livre. A fração residual insolúvel em acetona e em n-hexano, o EPDM-g-SAN, foi separada por filtração, seca e pesada, recebendo o nome de Fração2. A solução de EPDM em n-hexano, foi evaporada, e o EPDM foi seco e pesado, sendo denominado Fração 3. A Figura 1 mostra uma representação esquemática do procedimento experimental.

\section{Caracterização do AES e suas frações}

Os espectros na região de infravermelho foram obtidos no espectrômetro de infravermelho Bomen MB-Séries modelo MB-100, através da técnica de filme sobre janela de $\mathrm{NaCl}$. A faixa espectral analisada foi de $600 \mathrm{a} 4000 \mathrm{~cm}^{-1}$ com dezesseis acumulações e resolução de $2 \mathrm{~cm}^{-1}$.

As análises de ressonância magnética nuclear de hidrogênio $\left(\mathrm{RMN}^{1} \mathrm{H}\right)$ e carbono $13,\left(\mathrm{RMN}^{13} \mathrm{C}\right)$ foram realizadas no espectrômetro de ressonância magnética nuclear Varian Gemini 2000, com campo $\beta=7,05$ Tesla. Os espectros foram obtidos com pulso de $90^{\circ}$. O tempo de espera entre os pulsos, delay, para a análise de $\mathrm{RMN}^{13} \mathrm{C}$ foi de $20 \mathrm{~s}$ e o número de acumulações foi 256. As análises foram realizadas à temperatura ambiente em benzeno deuterado para o EPDM e em clorofórmio deuterado para o SAN. Utilizou-se aproximadamente 50 e $150 \mathrm{mg}$ de EPDM ou SAN para as análises de $\mathrm{RMN}^{1} \mathrm{H}$ e $\mathrm{RMN}{ }^{13} \mathrm{C}$, respectivamente.

A análise elementar do AES e suas frações foi realizada no equipamento CHNS/O Series II 2400 da Perkin Elmer, com temperatura da coluna de combustão e redução de $925^{\circ} \mathrm{C}$ e $640{ }^{\circ} \mathrm{C}$, respectivamente. A análise por calorimetria diferencial 
de varredura do AES e do resíduo da extração do SAN, foi realizada no equipamento MDSC-2910 da TA Intruments. Os experimentos foram conduzidos em atmosfera de argônio (fluxo de $50 \mathrm{~cm}^{3} / \mathrm{min}$ ) segundo o seguinte programa de análise: as amostras foram aquecidas a $160{ }^{\circ} \mathrm{C}$, deixadas em isoterma por 5 min, então foram resfriadas a $-80{ }^{\circ} \mathrm{C}$ e aquecidas até $160{ }^{\circ} \mathrm{C}$. A taxa de aquecimento e resfriamento foi de $20^{\circ} \mathrm{C} /$ min e a massa de amostra foi de aproximadamente $10 \mathrm{mg}$. As curvas obtidas foram normalizadas dividindo-se o fluxo de calor pela massa da amostra.

\section{Resultados e Discussão}

O método de extração seletiva, descrito neste trabalho, baseia-se na solubilidade diferenciada dos polímeros. De acordo com Morimoto ${ }^{[9]}$ o EPDM que apresenta parâmetro de solubilidade $\delta=8,0\left(\mathrm{cal} / \mathrm{cm}^{3}\right)^{1 / 2}$ é solúvel em solventes com baixo parâmetro de solubilidade, como: benzeno $(\delta=9,2$ (cal/ $\left.\left.\mathrm{cm}^{3}\right)^{1 / 2}\right)$, tolueno $\left(\delta=8,9\left(\mathrm{cal} / \mathrm{cm}^{3}\right)^{1 / 2}\right)$, heptano $(\delta=7,4(\mathrm{cal} /$ $\left.\left.\mathrm{cm}^{3}\right)^{1 / 2}\right)$ e hexano $\left(\delta=7,3\left(\mathrm{cal} / \mathrm{cm}^{3}\right)^{1 / 2}\right)$, enquanto que o SAN apresenta parâmetro de solubilidade dependente do teor de acrilonitrila, estando entre 9.3 e $\left.12\left(\mathrm{cal} / \mathrm{cm}^{3}\right)^{1 / 2}\right)$, apresentando solubilidade em solventes com alto parâmetro de solubilidade, como: dicloroetano $\left(\delta=9,76\left(\mathrm{cal} / \mathrm{cm}^{3}\right)^{1 / 2}\right)$, acetato de etila $\left(\delta=9,1\left(\mathrm{cal} / \mathrm{cm}^{3}\right)^{1 / 2}\right)$, dioxano $\left(\delta=9,9\left(\mathrm{cal} / \mathrm{cm}^{3}\right)^{1 / 2}\right)$, acetona $\left(\delta=9,9\left(\mathrm{cal} / \mathrm{cm}^{3}\right)^{1 / 2}\right)$ e acetonitrila $\left(\delta=11,9\left(\mathrm{cal} / \mathrm{cm}^{3}\right)^{1 / 2}\right)^{[19]}$. O AES por ser composto de EPDM e SAN é solúvel em cloro-
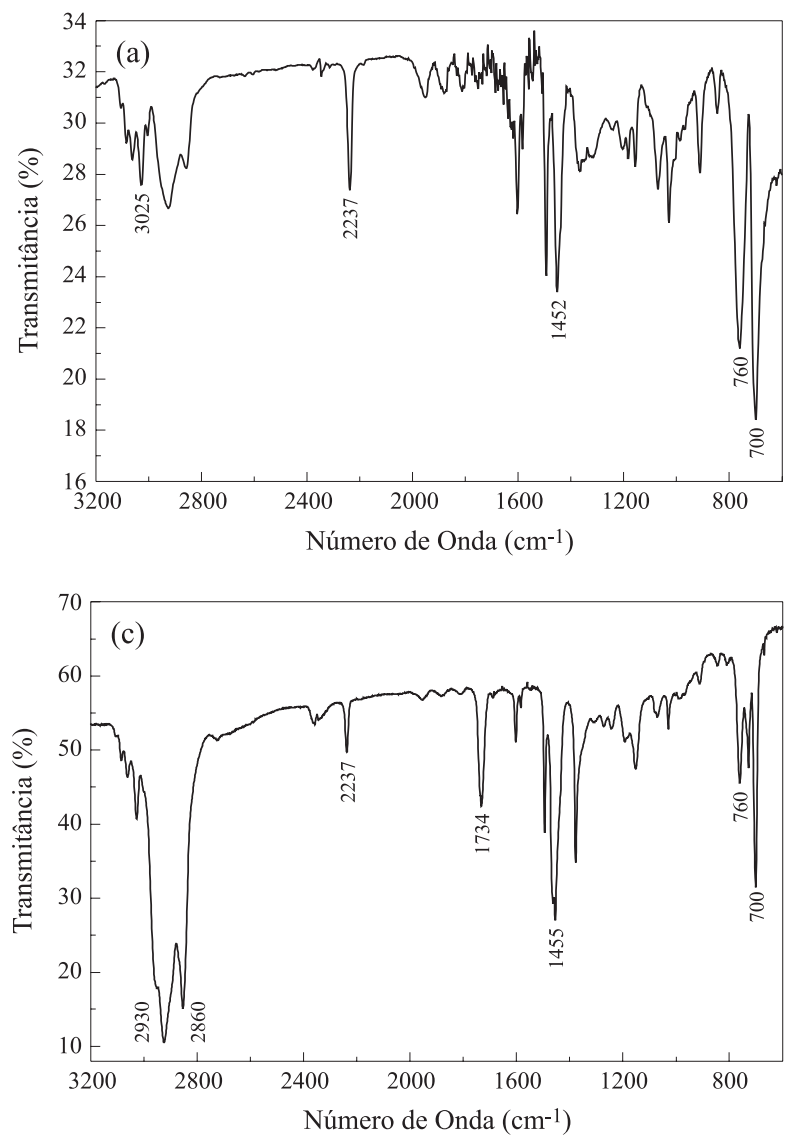

fórmio a temperatura ambiente. Sendo assim, para a extração seletiva da fase SAN do AES utilizou-se a acetona, enquanto que a fase EPDM foi extraída com n-hexano.

A utilização da espectroscopia na região do infravermelho, IV, é uma ferramenta prática e eficiente para a caracterização de grupos funcionais. Sendo assim, utilizou-se esta técnica para caracterizar as frações obtidas por extração seletiva. O espectro de IV correspondente à Fração 1, o SAN livre, Figura 2(a), apresenta uma banda referente ao estiramento do grupo $\mathrm{C} \equiv \mathrm{N}$ em $2237 \mathrm{~cm}^{-1}$, referente a acrilonitrila, uma banda em $3025 \mathrm{~cm}^{-1}$ atribuída ao estiramento $\mathrm{C}-\mathrm{H}$ do anel aromático do estireno, uma banda intensa em $1452 \mathrm{~cm}^{-1}$ atribuída à deformação angular do grupo $\mathrm{CH}_{2}$ e bandas características de anel aromático monosubstituído em 700 e $760 \mathrm{~cm}^{-1}$ dentre outras ${ }^{[2,10]}$. O espectro de IV da Fração 3, o EPDM, Figura 2(b), é característico de uma poliolefina, com uma banda em $1377 \mathrm{~cm}^{-1}$ atribuída à deformação simétrica do grupo $\mathrm{CH}_{3}$, uma banda intensa em $1464 \mathrm{~cm}^{-1}$ atribuída à deformação angular do grupo $\mathrm{CH}_{2}$ e duas bandas intensas em 2860 e $2960 \mathrm{~cm}^{-1}$ referentes aos estiramentos simétricos e assimétricos dos grupos $\mathrm{CH}_{2} \mathrm{e} \mathrm{CH}_{3}{ }^{[10]}$. O espectro da Fração 2, o EPDM-g-SAN, Figura 2 (c), apresenta tanto as bandas características do SAN como as do EPDM. A Figura 2(d) apresenta espectro do AES, também com as bandas características do EPDM e do SAN. A análise por espectroscopia de infravermelho permitiu concluir que a extração seletiva resultou em frações diferenciadas.
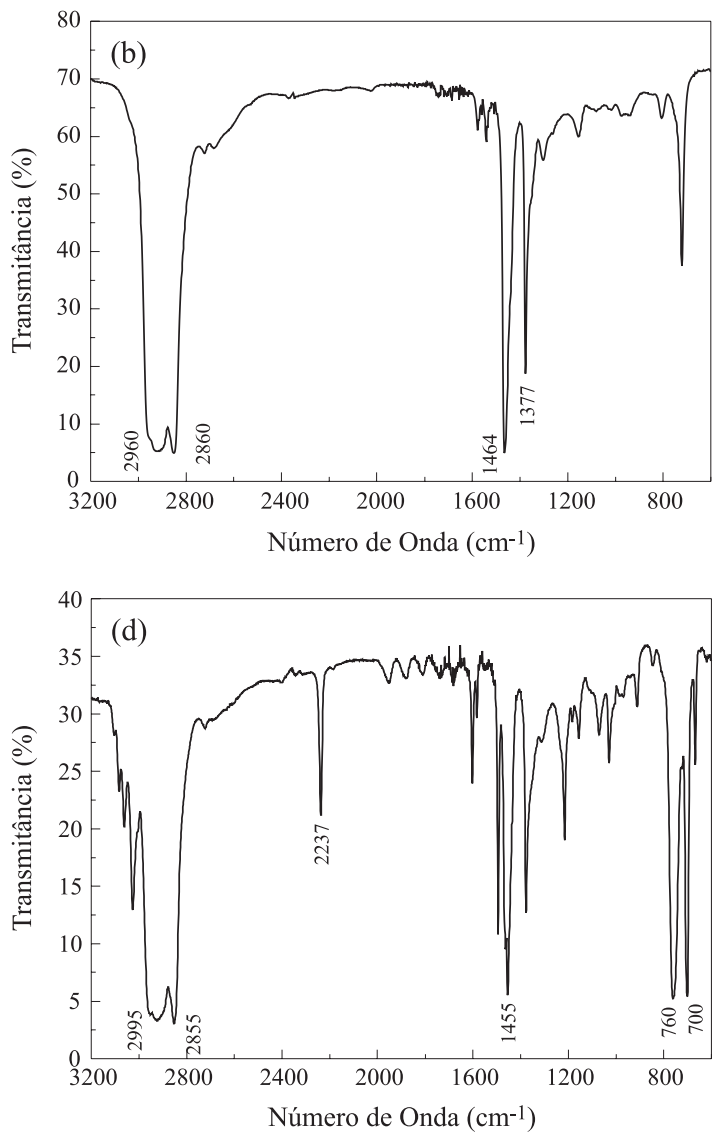

Figura 2. Espectros na região de infravermelho para: (a) SAN; (b) EPDM; (c) EPDM-g-SAN; (d) AES. Resolução de $2 \mathrm{~cm}^{-1}, 16$ varreduras. 
A quantidade das frações SAN, EPDM e EPDM-g-SAN isoladas na extração seletiva foi determinada gravimetricamente, sendo os resultados apresentados na Tabela 2. Através do teor de nitrogênio obtido da análise elementar da fração SAN determinou-se o teor de acrilonitrila, AN, assumindo-se que o SAN enxertado no EPDM tem a mesma composição do SAN livre. Esta consideração se baseia no fato de que a composição do SAN é determinada pelas razões de reatividade do estireno e acrilonitrila nas condições de síntese, polimerização radicalar em meio viscoso. Sabe-se que as razões de reatividade do estireno e acrilonitrila na copolimerização em massa são 0,41 e $0,04^{[20]}$, respectivamente. Embora o meio reacional afete os valores de razão de reatividade, em princípio as cadeias de SAN livre e enxertadas no EPDM não devem apresentar composição distinta, pois ela é determinada pelas razões de reatividade. Sendo assim, a partir do teor de acrilonitrila no SAN determinou-se o teor de SAN no EPDM-g-SAN e no AES. A fração de EPDM foi obtida subtraindo-se o teor de SAN do total.

Dependendo do fabricante, o EPDM pode apresentar um teor de dieno variando entre 1 e $5 \%$, e diferentes tipos de dieno: o 1,4-hexadieno, o diciclopentadieno ou o 2-etilideno5-norboneno, $\mathrm{ENB}^{[21]}$. Em alguns casos o teor de dieno no EPDM pode chegar até $9 \%{ }^{[12]}$. As diferenças de composição no EPDM têm grande influência na degradação térmica. Por exemplo, o aumento do teor de etileno ou a diminuição no teor do dieno ENB causam um aumento na estabilidade térmica do $\mathrm{EPDM}^{[22,23]}$.

Um método geralmente utilizado para determinar a composição molar do EPDM é a espectroscopia de infravermelho, pelo método ASTM D 3900. Contudo, a aplicação deste método requer a utilização de padrões. Uma alternativa para determinação da composição do EPDM é a ressonância magnética nuclear. $\mathrm{O}$ espectro de $\mathrm{RMN}{ }^{1} \mathrm{H}$ da Fração 3, o EPDM, apresentado na Figura 3(a), é típico de um hidrocarboneto, apresentando a maioria dos picos em uma

Tabela 2. Composição mássica do AES e de suas frações

\begin{tabular}{|c|c|}
\hline AES & $\begin{array}{c}22 \% \text { SAN }^{(\mathrm{a}, \mathrm{d})}, \\
13 \% \%^{(\mathrm{a})} \text { EPDM, } \\
65 \%^{(\mathrm{a})} \text { EPDM-g-SAN }\end{array}$ \\
\hline EPDM livre & $\begin{array}{c}\text { 68,9\% } \% \text { polietileno }{ }^{(\mathrm{c})} \\
26,5 \% \text { polipropileno }^{(\mathrm{c})} \\
\text { 4,6\% } \text { dieno }^{(\mathrm{c})}\end{array}$ \\
\hline SAN livre & $\begin{array}{c}28,0 \% \mathrm{AN}^{(\mathrm{c})} \\
31 \% \mathrm{AN}^{(\mathrm{b})}\end{array}$ \\
\hline EPDM-g-SAN & $\begin{array}{c}24,1 \% \mathrm{SAN}^{(\mathrm{b})} \\
75,9 \% \mathrm{EPDM}^{(\mathrm{b})}\end{array}$ \\
\hline
\end{tabular}

(a) Extração/gravimetria

(b) Análise Elementar

(c) $\mathrm{RMN}^{13} \mathrm{C}$

(d) DSC estreita faixa de deslocamento químico, entre 0 e 3,0 ppm. Na Figura 3(b) observa-se uma ampliação da região entre 5,6 e 4,5 ppm, na qual verifica-se os picos em 5,17 e 5,42 ppm, que são característicos do dieno 2-etilideno-5norboneno, ENB. Os picos são atribuídos à ressonância das conformações $E$ e $Z$ do ENB. Estas conformações denotam a posição de grupos químicos em relação à dupla ligação. A conformação é chamada $E$ quando os grupos de maior prioridade estão do mesmo lado da dupla ligação ${ }^{[24]}$. Por exemplo, a molécula de ENB apresentada na Figura 3(a), representa a conformação $E$ do dieno que recebe este símbolo estereoquímico porque o grupo $\mathrm{CH}_{3}$ (carbono 9) apresenta maior prioridade que o $\mathrm{H}$, ligado ao carbono 8 . No espectro da Figura 3(b) observam-se os picos em 5,42 e 5,17 ppm, sendo o isômero do ENB, apresentado na Figura 3(a), o responsável pelo pico em 5,17 $\mathrm{ppm}^{[25]}$.

A razão entre a integral destes picos, que neste caso é 3 , corresponde a razão destes isômeros encontrados nos polímeros comerciais ${ }^{[26]}$. Na região de 0 a 3,5 ppm encontram-se os picos relativos aos hidrogênios do etileno, do propileno e da região alifática do ENB.

O teor de ENB no EPDM pode ser determinado subtraindo-se do valor da integral da região do espectro onde ocorrem os sinais referentes a hidrogênios de segmentos alifáticos do EPDM, 0 a 3,5 ppm, da contribuição relativa aos hidrogênios alifáticos do ENB, utilizando-se a Equação 1. A equação

(a)
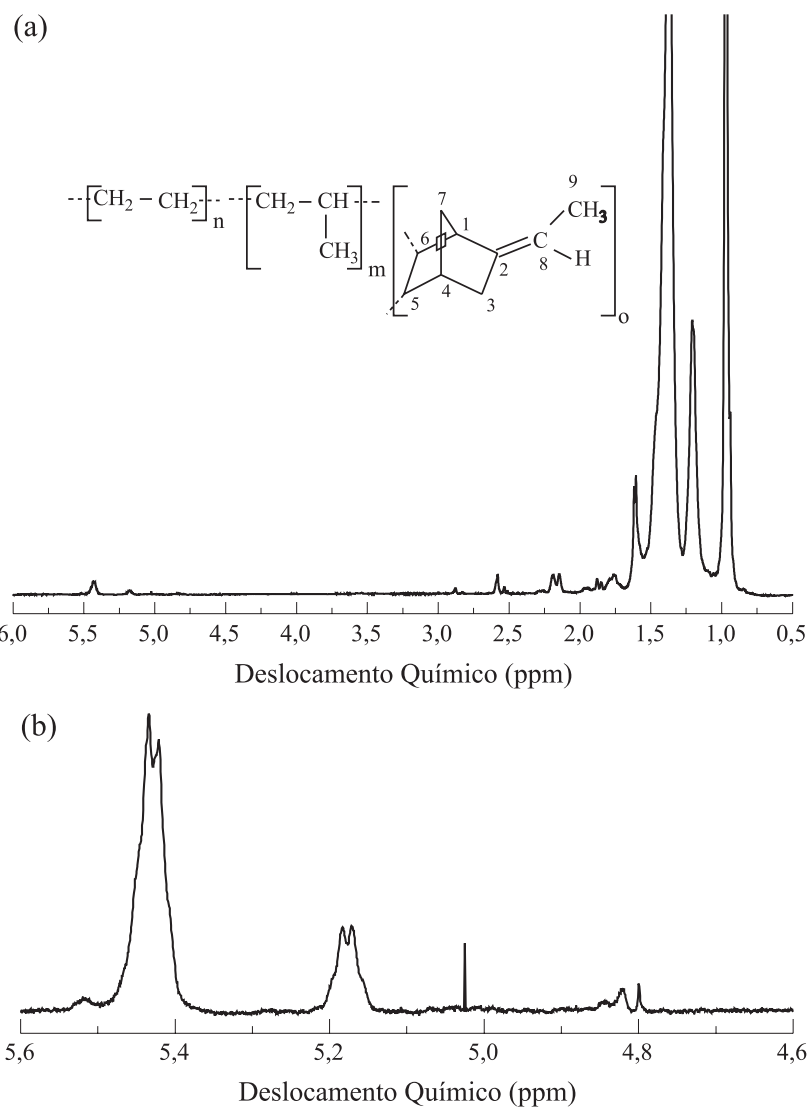

Figura 3. Espectro de RMN ${ }^{1} \mathrm{H}$ da fração de EPDM extraído do AES. (a) todo o espectro; (b) ampliação da região olefínica característica do dieno ENB, 303 acumulações. 
considera a massa molar do ENB, $120 \mathrm{~g} / \mathrm{mol}$ e a massa de uma unidade repetitiva $\mathrm{CH}_{2}, 14 \mathrm{~g} / \mathrm{mol}$.

$$
\operatorname{ENB}(\% \text { massa })=\frac{d * 120}{d * 120+\frac{1}{2}(a-11 d) * 14} * 100
$$

sendo: $a$ é a integral da região alifática e $d$ a integral da região olefínica, entre 5 e 6 ppm ${ }^{[27]}$.

$\mathrm{O}$ teor de ENB obtido aplicando-se a Equação 1 foi de $4,6 \%$ em massa, estando dentro da faixa esperada para um produto industrial.

A Figura 4 apresenta o espectro de $R M N^{13} \mathrm{C}$ do EPDM utilizado para a determinação da razão etileno/propileno do EPDM. O teor de etileno foi calculado segundo a Equação 2 ${ }^{[27,28]}$ :

$$
\% \text { em mol de etileno }=\frac{(\mathrm{S}+\mathrm{T}-2 \mathrm{P})}{\mathrm{S}+\mathrm{T}} 100
$$

sendo: $\mathrm{P}, \mathrm{S}$ e $\mathrm{T}$ as áreas dos picos referentes aos carbonos primários, secundários e terciários, respectivamente.

A atribuição dos picos do espectro de $\mathrm{RMN}^{13} \mathrm{C}$, Tabela 3, foi feita segundo a literatura. Os deslocamentos químicos são ligeiramente diferentes dos apresentados na literatura, devido à utilização do benzeno deuterado como solvente, que propiciou a realização da análise em temperatura ambiente, ao invés do tetracloroetano ou orto-diclorobenzeno à $125^{\circ} \mathrm{C}^{[27,28]}$.

A nomenclatura utilizada na Tabela 3 está de acordo com

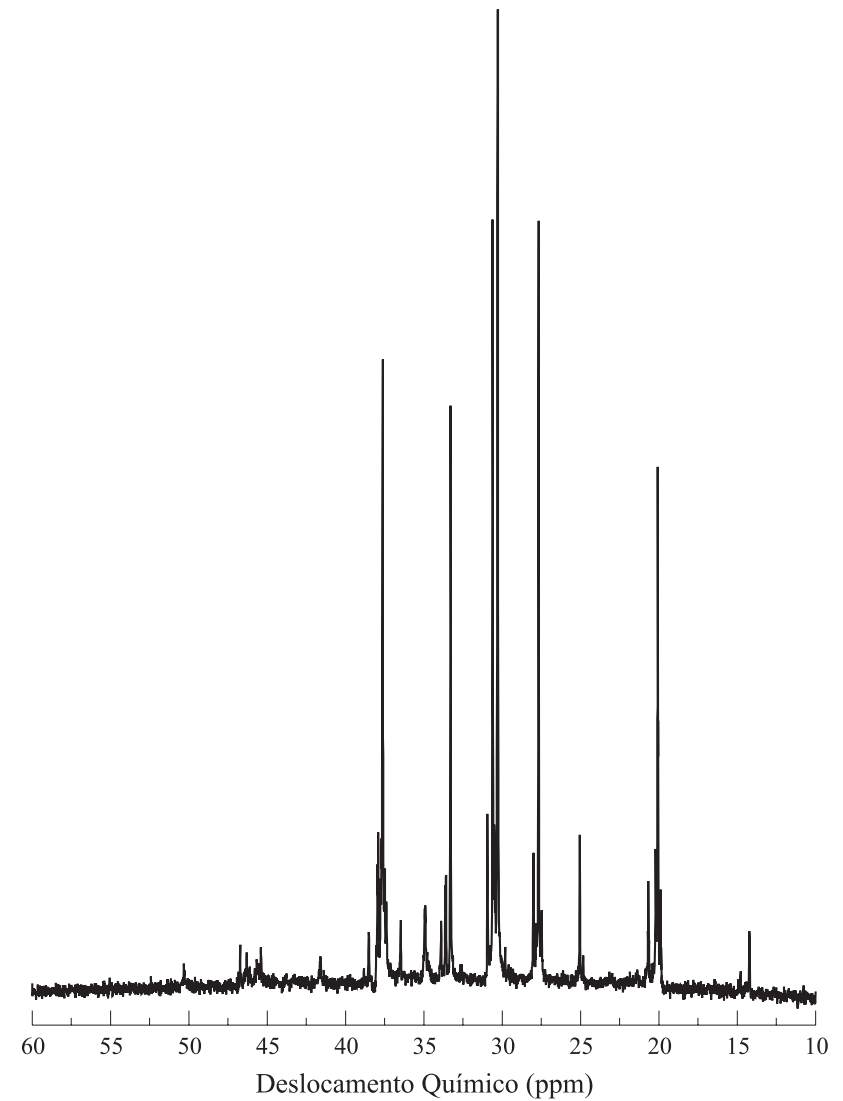

Figura 4. Espectro de $\mathrm{RMN}^{13} \mathrm{C}$ do EPDM extraído com n-hexano, 256 acumulações.
Tabela 3. Segmento, limite de integracão e área relativa aos picos do EPDM, obtidos a partir do espectro de $\mathrm{RMN}^{13} \mathrm{C}$

\begin{tabular}{lcc}
\hline \multicolumn{1}{c}{ Segmento } & $\begin{array}{c}\text { Limite de integração } \\
(\mathbf{p p m})\end{array}$ & Área \\
\hline $\mathrm{ENB}\left(\mathrm{C}_{1 \mathrm{E}}\right)$ & $52,00-50,00$ & 0,3 \\
$\mathrm{~S}_{\alpha \alpha}+\mathrm{ENB}\left(\mathrm{C}_{5}+\mathrm{C}_{6}\right)+\mathrm{C}_{1 \mathrm{Z}}$ & $48,50-44,50$ & $1 / 0,27^{*}$ \\
$\mathrm{ENB}\left(\mathrm{C}_{4}\right)$ & $43,00-41,00$ & 0,4 \\
$\mathrm{~S}_{\alpha \gamma}+\mathrm{S}_{\alpha \delta}$ & $39,40-36,85$ & 2,4 \\
$\mathrm{ENB}\left(\mathrm{C}_{3 \mathrm{E}}\right)$ & $36,85-36,20$ & - \\
$\mathrm{S}_{\alpha \beta}$ & $36,20-34,30$ & 1,6 \\
$\mathrm{~T}_{\gamma \gamma}+\mathrm{T}_{\gamma \delta}+\mathrm{ENB}\left(\mathrm{C}_{7}\right)$ & $34,29-33,47$ & $1,3 / 0,98^{*}$ \\
$\mathrm{~T}_{\delta \delta}+\mathrm{T}_{\beta \gamma}+\mathrm{T}_{\beta \delta}+\mathrm{S}_{\gamma}$ & $33,47-30,61$ & 0,4 \\
$\mathrm{~S}_{\gamma \delta}+\mathrm{S}_{\delta \delta}$ & $30,61-29,32$ & 2,8 \\
$\mathrm{~S}_{\beta \gamma}+\mathrm{S}_{\beta \delta}$ & $28,22-26,63$ & 1,6 \\
$\mathrm{~S}_{\beta \beta}$ & $25,60-23,95$ & 0,6 \\
$\mathrm{P}_{\beta \beta}+\mathrm{P}_{\beta \gamma}+\mathrm{P}_{\beta \delta}$ & $22,50-19,00$ & 1,5 \\
$\mathrm{ENB}_{(\mathrm{C}}\left(\mathrm{C}_{9}\right)$ & $15,00-13,00$ & 0,5 \\
\hline
\end{tabular}

* Área corrigida de acordo com a equação (3)<smiles>CCCCC(C)C(C)(C)C(C)(C)C(CC)CC</smiles>

Figura 5. Nomenclatura dos carbonos utilizada na Tabela 2.

Carman e cols. ${ }^{[29]}$, Smith ${ }^{[30]}$ e Cheng ${ }^{[31]}$. As letras gregas designam a distância de carbonos em relação a um carbono terciário, $\mathrm{CH}$, em ambas as direções da cadeia. Por exemplo, se um carbono secundário $\alpha \alpha$ se encontra entre dois carbonos terciários, é designado $\mathrm{S}_{\alpha \alpha}$. $\mathrm{O}$ carbono designado como terciário $\mathrm{T}_{\beta \gamma}$ está a um carbono de distância de outro carbono terciário e a dois carbonos de distância de um terceiro, como exemplificado na Figura 5.

Como na integração dos picos é considerada também a contribuição do dieno, ENB, pode-se descontar esta contribuição através da utilização da equação 3 , na qual os índices se referem ao número do carbono do isômero " $E$ " [27].

$$
\begin{gathered}
{[\mathrm{ENB}]=1 / 4\left(4 / 3 \mathrm{C}_{1 \mathrm{E}}+\mathrm{C}_{4}+4 / 3 \mathrm{C}_{3 \mathrm{E}}+\mathrm{C}_{9}\right)} \\
\mathrm{S}^{\prime} \alpha \alpha=\mathrm{S} \alpha \alpha-2,25[\mathrm{ENB}] \\
\mathrm{e} \\
(\mathrm{T} \gamma \gamma+\mathrm{T} \gamma \delta)^{\prime}=\mathrm{T} \gamma \gamma+\mathrm{T} \gamma \delta-[\mathrm{ENB}]
\end{gathered}
$$

sendo que: $[\mathrm{ENB}]$ denota o valor da integral.

A Tabela 3 mostra os valores de área e respectivos limite de integração.

$\mathrm{O}$ teor de polietileno, calculado pela equação 2 e corrigido pela equação 3, para o EPDM foi de $68,9 \%$. O teor de polipropileno no EPDM pode ser obtido subtraindo-se de $100 \%$ a porcentagem de etileno, $68,9 \%$ e de dieno, $4,6 \%$, sendo assim, o teor de polipropileno é $26,5 \%$. 
$\mathrm{Na}$ copolimerização radicalar de estireno e acrilonitrila a razão de reatividade dos monômeros são respectivamente 0,41 e $0,04^{[20]}$, indicando que o estireno é muito mais reativo que a acrilonitrila. Segundo Qu e cols. ${ }^{[14]}$ a razão em massa de estireno e acrilonitrila de 3,13 corresponde ao ponto azeotrópico, ou seja, a composição mássica na qual a razão entre os monômeros é a mesma no meio racional e no copolímero formado, sendo assim, próximo a esta composição a probabilidade de formar homopolímeros é baixa.
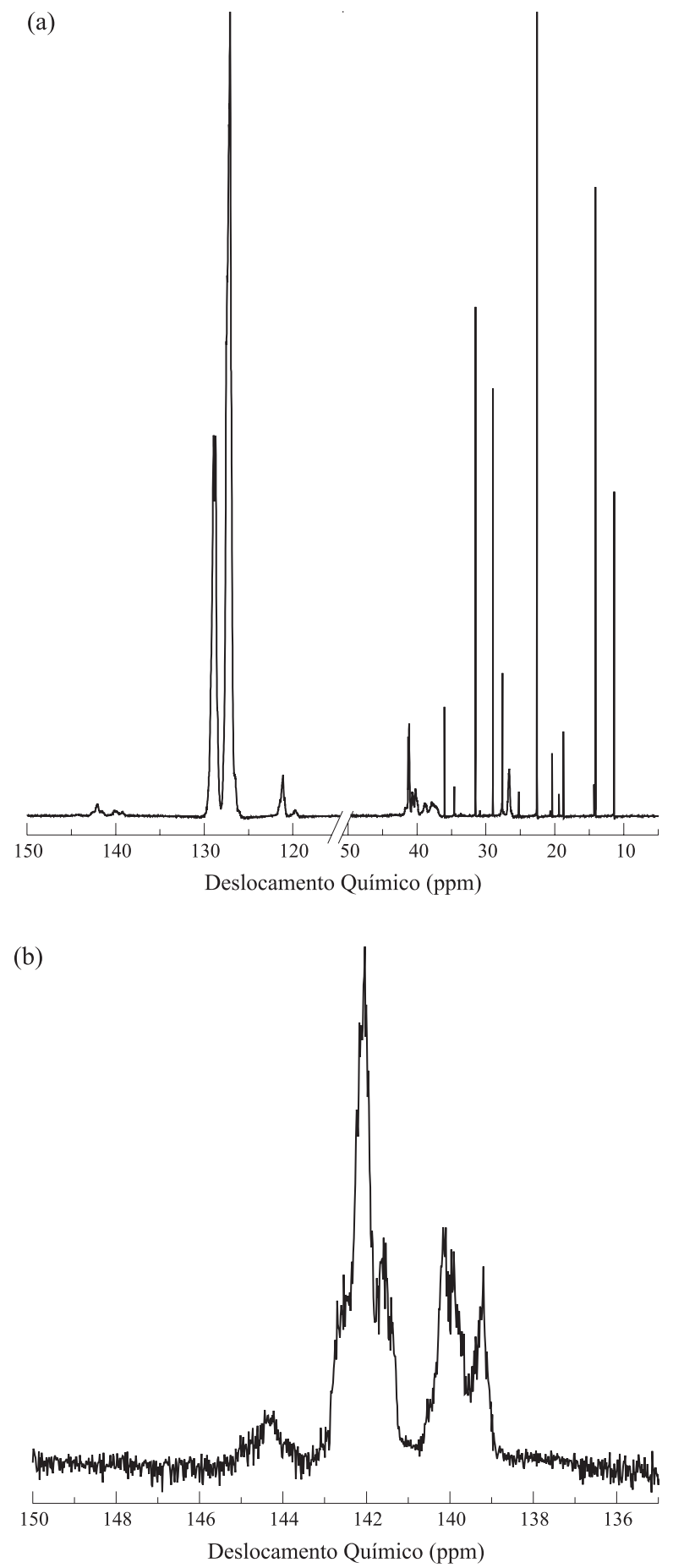

Figura 6. Espectro de $\mathrm{RMN}^{13} \mathrm{C}$ da fase $\mathrm{SAN}$ extraída do AES. (a) todo o espectro; (b) ampliação da região das tríades centradas no estireno.
A fase SAN extraída do AES foi caracterizada quanto à sua composição por $\mathrm{RMN}^{13} \mathrm{C}$, como apresentado na Figura 6 (a). No espectro foi retirada a região de 110 a 50 ppm, na qual tem-se o pico do solvente utilizado. Os picos acima de 135 ppm, destacados na Figura 6(b), são relativos as tríades centradas no estireno. São elas: SSS = 145 a 143 ppm; SSA e ASS $=143$ a 141 ppm e ASA $=140$ a 138 ppm. De 130 a 125 ppm encontram-se os picos relativos aos carbonos ligados ao anel aromático do estireno. As tríades centradas na acrilonitrila são: $\mathrm{SAS}=122$ a $120 \mathrm{ppm}$; SAA ou AAS $=120$ a 119 ppm. Não foram observadas as tríades AAA que apareceriam abaixo de 119 ppm, o que é conseqüência da diferença de reatividade da acrilonitrila em relação ao estireno, que torna difícil a ocorrência de homopolímeros de acrilonitrila ${ }^{[32,33]}$. A ocorrências das tríades tanto centradas no estireno como na acrilonitrila é uma evidência que o processo de copolimerização foi aleatório. A porcentagem de acrilonitrila obtida baseando-se na área das tríades centradas na acrilonitrila e na área dos picos relativos ao carbono ligado ao anel aromático foi de aproximadamente $28 \%$. Este valor é próximo ao determinado por análise elementar, e daquela fornecida pelo fabricante ${ }^{[18]}$.

A separação e caracterização química dos componentes do AES mostrou que se trata de um material quimicamente complexo. Para elucidar o comportamento de fases foi realizada análise de calorimetria diferencial de varredura. As curvas DSC normalizadas com respeito a massa, para o AES e para o resíduo sólido da extração de SAN (EPDM- $g$-SAN e EPDM) são apresentadas na Figura 7.

As curvas de DSC apresentam duas transições vítreas, tanto para o AES, como para a mistura (EPDM-g-SAN e EPDM). A transição vítrea em $-43^{\circ} \mathrm{C}$ é atribuída à fase EPDM, enquanto que em $113{ }^{\circ} \mathrm{C}$ verifica-se a transição vítrea da fase SAN.

Uma forma alternativa para determinação da composição das frações extraídas do AES é a analise da variação da capacidade calorífica, $\Delta \mathrm{Cp}$, referentes às transições vítreas de ambas as fases, obtidos a partir das curvas de DSC. O $\Delta \mathrm{Cp}$ reflete a variação nos graus de liberdade de um material e está relacionado com o ganho de flexibilidade das cadeias

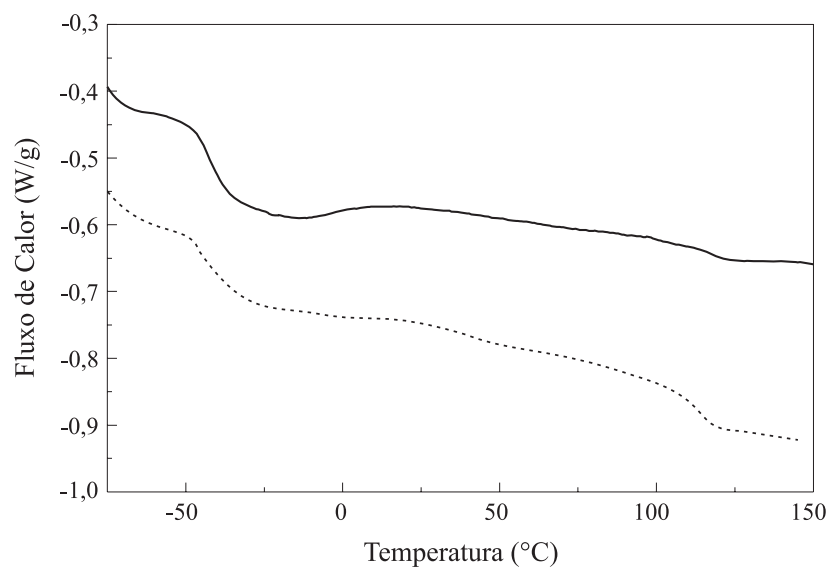

Figura 7. Curvas de DSC para: (….) AES; (-) Fração 2 (EPDM-g-SAN + EPDM). Razão de aquecimento de $20{ }^{\circ} \mathrm{C} / \mathrm{min}$, fluxo de argônio $\left(50 \mathrm{~cm}^{3} /\right.$ $\min )$. 
poliméricas quando passam do estado vítreo para o líquido. Comparando-se as curvas de DSC para o AES e para a mistura, obtida após a extração do SAN, verifica-se que ocorreu um aumento do $\triangle \mathrm{Cp}$ da fase EPDM, passando de $0,25 \mathrm{~J} / \mathrm{g} .{ }^{\circ} \mathrm{C}$ para o AES a $0,33 \mathrm{~J} / \mathrm{g} .{ }^{\circ} \mathrm{C}$ para a mistura. Simultaneamente ocorre uma diminuição no $\Delta \mathrm{Cp}$ da fase $\mathrm{SAN}$, passando de 0,18 para $0,10 \mathrm{~J} / \mathrm{g} .{ }^{\circ} \mathrm{C}$, respectivamente. Como o $\Delta \mathrm{Cp}$ da fase SAN do EPDM-g-SAN é $\sim 56 \%$ do valor de $\Delta \mathrm{Cp}$ da mesma fase no AES, esta diferença de $44 \%$ corresponderia ao teor de SAN extraído. Por outro lado, o AES possui $50 \%$ de SAN, levando à conclusão que foi extraído aproximadamente $22 \%$ do SAN e que aproximadamente $28 \%$ de SAN está enxertado no EPDM. Pode-se notar que o teor de AES enxertado no EPDM obtido por DSC é muito semelhante aos dados obtidos pela análise elementar (Tabela 2), e pelo fabricante (Tabela 1), mostrando a validade desta análise neste caso.

\section{Conclusões}

Partindo-se do AES, um material complexo, composto de EPDM- $g$-SAN , EPDM livre e SAN livre, foi possível isolar os diferentes componentes por extração seletiva e caracterizar quimicamente as várias frações. Os resultados obtidos para a composição global do AES são concordantes com as informações do fornecedor do AES, mostrando que a metodologia adotada no presente trabalho é adequada para a caracterização de um material complexo como o AES.

\section{Agradecimentos}

Os autores agradecem à FAPESP pelo apoio financeiro (processo $\mathrm{n}^{\circ}$ 03/01756-0) e a Compton Corporation pela doação do AES.

\section{Referências Bibliográficas}

1. Park, D. J.; Ha, C. S.; Cho, W. J. J. Appl. Polym. Sci. 67 1345 (1998).

2. Park, D. J.; Ha, C. S.; Cho, W. J. J. Appl. Polym. Sci. 54763 (1994).

3. Larocca, N.M.; Hage, E.Jr.; Pessan, L.A. Polymer 45, 5265 (2004).

4. Qu, X.; Shang, S.; Liu, G.; Zhang, S. Zhang, Y.; Zhang, L. J. Appl. Polym. Sci. 91, 1685 (2004).

5. Mukerjee, A. K. e Gupta, B. D. J. Macroml. Sci. Chem., A19, 1069 (1983).

6. Zeng, Z.; Wang, L.; Cai, T.; Zeng, X.; J. Appl. Polym. Sci. 94, 416 (2004).

7. Bassani, A.; Pessan, L. A.; Hage Jr, E. Polímeros: Ciência e Tecnologia 12, 102 (2002).

8. Chiantore, O.; Lazzari, M.; Ravanetti, G. P.; Nocci, R. J. Appl. Polym. Sci.: Appl. Polym. Symp. 51, 249 (1992).

9. Morimoto, M. J. Appl. Polym. Sci 26, 261 (1981).

10. Bae, Y. O.; Ha, C. S.; Cho, W. J. Eur. Polym J. 27, 121 (1991).
11. Chiantore, O.; Lazzari, M.; Guaita, M. Polym. Bull.,34 3 (1995).

12. Chiantore, O Ind. Eng. Chem. Res. 36, 1276 (1997).

13. Lu, M.; Keskkula, H.; Paul, D. R. J. Appl. Polym. Sci 58, 1775 (1995).

14. Qu, X.; Shang, S.; Liu, G.; Zhang, L. J. Appl. Polym. Sci. 86, 428 (2002).

15. Bassani, A.; Machado, A.V.; Covas, J.A.; Hage, E.; Pessan, L.A. Anais do $18^{\circ}$ Polymer Progress Society Portugal, (2002).

16. Guidoni, D.; Fasulo, G. C.; Cecchele, D.; Merlotti, M.; Sterzi, G.; Nocci, R. J. Mat. Sci. 284119 (1993).

17. Turchette, R. Dissertação de Mestrado, Universidade Estadual de Campinas, 2002.

18. Royaltuf Modified EPDM for Tougher Engineering Thermoplastics and other Rubber and Plastics Aplplication, Umiroyal Chemical, 1992.

19. Brandrup, J.; Immergut, E.H. Polymer Handbook, $2^{\circ}$ ed, John Wiley \& Sons, New York, 1975.

20. Carraher Jr, C.E.; Seymour Carraher'S. Polymer Chemistry, an Introduction, $4^{\circ}$ ed, Marcel Dekker; New York, 1996.

21. De Paoli, M-A.; Geuskens, G. Polym. Degrad. Stabil. 21, 277 (1988).

22. Gamlin, C.; Dutta, N.; Choudhury, N. R, Kehoe, D.; Matisons, J. Therm. Acta 367-368, 185 (2001).

23. Delor, F.; Teissedre, G.; Baba, M.; Lacoste, J. Polym. Degrad. Stabil. 60, 321 (1998).

24. Streitwieser, A.; Heathcock, C.H.; Kosower, E.M. "Introduction to Organic Chemistry" $4^{\circ}$ ed, Macmillan Publishing; New York, 2002.

25. Chiantore, O.; Cinquina, P.; Guaita, M. Eur. Polym. J. 30, 1043 (1994).

26. Winters, R.; Heinen, W.; Verbruggen, M. A. L.; Lugtenburg, J.; van Duin, M.; de Groot, H. J. M. Macromolecules, 35, 1958 (2002).

27. Kolbert, A. C.; Didier, J. G., J. Appl. Polym. Sci. 71, 523 (1999).

28. Di Martino, S.; Kelchtermans, M., J. Appl. Polym. Sci. 56, 1781 (1995).

29. Carman, C. J.; Harrington, R. A.; Wilkes, C. E., Macromolecules. 10, 536 (1977).

30. Smith, W. V. J. Polym. Sci. Polym. Phys. 18, 1587 (1980).

31. Cheng, H. N. Macromolecules 17, 1950 (1984).

32. Carraher Jr, C.E.; Seymour Carraher'S. Polymer Chemistry, an Introduction, $4^{\circ}$ ed, Marcel Dekker; Inc Nova Iorque, 19996.

33. Sanghvi, P.G.; Patel, A. C.; Gopalkrishnan, K. S.; Devi, S. Eur. Polym. J. 362275 (2000).

Enviado: 12/09/05

Reenviado: 23/01/06

Aprovado: 01/02/06 